\title{
Continuous 4 Percent Albumin Versus Intermittent 20 Percent Albumin in Adults with Septic Shock: A Prospective, Phase IV, Open-label Randomized Trial
}

\author{
Francis Schneider ${ }^{1,6}$, Vincent Castelain ${ }^{1}$, Guillaume Morel ${ }^{1}$, Anne-Florence Dureau ${ }^{2}$, \\ Antoine Poidevin ${ }^{2}$, Pierre-Olivier Ludes ${ }^{3}$, Thibaut Fabacher ${ }^{4}$, Bernard Senger ${ }^{6}$, Nicolas Meyer ${ }^{4,5}$, \\ Marie-Hélène Metz-Boutigue ${ }^{6, *}$ \\ ${ }^{1}$ Medical Intensive Care, Hautepierre Hospital I, University Hospital of Strasbourg, Federation of Translational Medicine, Faculty of \\ Medicine, University of Strasbourg, Strasbourg, France \\ ${ }^{2}$ Medical Intensive Care, E. Muller Hospital, Hospital Group Mulhouse-Sud Alsace, Mulhouse, France \\ ${ }^{3}$ Surgical Critical Care Department, Hautepierre Hospital II, Universitary Hospital of Strasbourg, Federation of Translational Medicine, \\ Faculty of Medicine, University of Strasbourg, Strasbourg, France \\ ${ }^{4}$ Group of Methods in Clinical Research, Public Health Department, Universitary Hospital of Strasbourg, Federation of Translational \\ Medicine, Faculty of Medicine, University of Strasbourg, Strasbourg, France \\ ${ }^{5}$ ICUBE, Laboratory of Engineer Science, Computer Science and Imagery, Faculty of Physic and Engineering, University of Strasbourg, \\ Illkirch, France \\ ${ }^{6}$ BioMaterials and BioEngineering, National Institute of Health and Medical Research, Faculty of Odontology, University of Strasbourg, \\ Strasbourg, France
}

\section{Email address:}

Francis.Schneider@chru-strasbourg.fr (F. Schneider), Vincent.Castelain@chru-strasbourg.fr (V. Castelain),

guillaume.morel@chru-strasbourg.fr (G. Morel), af.dureau@gmail.com (Anne-Florence D.), poidevina@ghrmsa.fr (A. Poidevin), pierreolivier-ludes@chru-strasbourg.fr (Pierre-Olivier L.), thibaut.fabacher@chru-strasbourg.fr (T. Fabacher), bernard.senger@inserm.fr (B. Senger), nicolas.meyer@chru-strasbourg.fr (N. Meyer), marie-helene.metz@inserm.fr (Marie-Hélène Metz-Boutigue)

${ }^{*}$ Corresponding author

\section{To cite this article:}

Francis Schneider, Vincent Castelain, Guillaume Morel, Anne-Florence Dureau, Antoine Poidevin, Pierre-Olivier Ludes, Thibaut Fabacher, Bernard Senger, Nicolas Meyer, Marie-Hélène Metz-Boutigue. Continuous 4 Percent Albumin Versus Intermittent 20 Percent Albumin in Adults with Septic Shock: A Prospective, Phase IV, Open-label Randomized Trial. American Journal of Internal Medicine. Vol. 8, No. 3, 2020, pp. 89-100. doi: 10.11648/j.ajim.20200803.11

Received: March 13, 2020; Accepted: March 30, 2020; Published: April 23, 2020

\begin{abstract}
Whether a specific way of infusing albumin affects outcome in patients with major oxidative stress remains uncertain. To determine whether outcome measurements (survival, organ failure and care-related infections) are different according to different regimens of albumin infusion, we conducted a phase IV, randomized, open-label trial to compare the effects of continuous infusion of 4 percent albumin versus intermittent 20 percent albumin on outcome measurements in three third level-hospital intensive care unit (ICU) patients with septic shock. We randomly assigned 125 consecutive patients with septic shock when serum albumin became $\leq 20 \mathrm{~g} / \mathrm{L}$. Patients received either 4 percent albumin $(12.5 \mathrm{~mL} / \mathrm{kg})$ continuously or 20 percent albumin $(100 \mathrm{~mL}$ over $1 \mathrm{~h} / 8 \mathrm{~h})$ intermittently (controls) until serum albumin ranged between 25 and $30 \mathrm{~g} / \mathrm{L}$ and norepinephrine could be weaned. The primary outcome measure was death from any cause during the 28-day period after randomization. The other outcome parameters were ICU- and hospital length of stay, serum albumin concentrations, SOFA score and lactate over the 4 days following inclusion, care-related infections and tolerance of albumin over the 28 -day period after randomization. Data were analyzed with Bayesian methods. Of the 125 patients who underwent randomization, 63 received 4 percent albumin and 62 received 20 percent albumin; groups had balanced baseline characteristics. There were 19 deaths in the experimental group, as compared with 20 in the control group $(\operatorname{Pr}=0.40)$. The proportion of patients with new multiple-organ failure (assessed by daily SOFA) was similar in the groups $(\mathrm{RR}=0.71$ [0.29-1.41], $\mathrm{Pr}=0.14)$. There were no differences in the medians [IQR]) numbers of days spent in the ICU $(12.0[7.5 ; 22.0]$ versus 13 [8.0; 24.5] days, Pr=0.23), in
\end{abstract}


days spent within hospital $(29.0$ [10.5; 44.0] versus 24 [14.0; 46.8] days, $\operatorname{Pr}=0.32)$. In contrast, there were fewer patients with care-related infection in the study group, $(14.3 \%$ versus $45.2 \%, \operatorname{Pr}<0.001)$. Limitations concern lack of double blinding related to different regimens of infusion: this may impact results. To conclude, the continuous supply of 4 percent albumin in septic shock patients with serum albumin $\leq 20 \mathrm{~g} / \mathrm{L}$ decreases care-related infection (by two third) but does not result in better survival.

Keywords: Albumin, Care-related Infection, Outcome, Oxidative Stress, Septic Shock

\section{Introduction}

Meta-analyses about resuscitation using albumin in critically ill patients at risk of complications have concluded that human albumin solutions were not robustly effective at reducing all-cause mortality [1-3]. Attention has mainly been paid to fluid resuscitation of shock. These reports have left unsolved issues related to non-oncotic properties of therapeutic albumin [4], among which are timing of albumin administration, amounts of albumin to deliver, and the concentrations of the drug required to impact positively on outcome. Well-designed studies suggest that: 1) an early timing of albumin improves microvascular perfusion [5]; 2) 4 percent albumin be better for early fluid resuscitation [3] and 20 percent albumin for maintaining normal serum albumin concentrations [6] and at improving oxygenation in ARDS (acute respiratory distress syndrome) patients as well [7]. Hyper-oncotic colloid solutions per se do not injure the kidney [8], whereas 4 percent albumin worsens prognosis in patients with traumatic brain injury [9]. Whether any form of therapeutic albumin protects in vivo against infection is suggested in some studies $[10,11]$, but the benefit of albumin in septic conditions seems of borderline importance [2].

Serum albumin is reported as having a powerful antioxidant activity [4] whose expression is related to the preservation of its structural and functional activity [12]. Oxidative stress present at the acute phase of critical illnesses deteriorates the functional activity of albumin and is associated with increased mortality $[13,14]$. We took into account that, in patients requiring volume resuscitation and norepinephrine for acute circulatory failure, endogenous albumin may no longer display proper antioxidant and scavenging activities, binding and transport of substances, regulation of endothelial function and of the systemic inflammatory response [15]. In this setting, acute and shortterm infusions of therapeutic albumin increase serum albumin concentrations, but whether - and how long exogenous albumin remains functionally efficient for nononcotic properties is unknown [16]. An antioxidant being "any substance that, when present at low concentration, compared with those of an oxidizable substrate, significantly delays or prevents oxidation of that substrate" [17], we set the hypothesis that a long lasting infusion of small amounts of albumin may permanently preserve some low amounts of non-oxidized antioxidant albumin available for physiological purposes rather than would do a large and acute supply. Our group has recently reported such a possibility for the restoration of activity of the CGA-derived peptide Vasostatin-I whose functional activity is lost by oxidation
[18]. Finally, a rapid supply of large amounts of albumin during full blown oxidative stress will also necessarily end up in large amounts of oxidized albumin potentially related to pathological conditions induction [4].

The objective of the present trial was to evaluate the effect of low and continuous 4 percent albumin supplementation as an anti-oxidant drug in critically ill patients with acute circulatory failure requiring the infusion of norepinephrine and developing acute albumin $\leq 20 \mathrm{~g} / \mathrm{L}$ when compared to a standard strategy of hyper oncotic albumin administration intermittently. Our a priori hypothesis was that this supplementation would impact on outcome. We present herein the final analysis of a randomized trial which was stopped for futility on mortality and for an ethical issue linked to unexpected good impact of the experimental treatment on a secondary outcome (carerelated infection occurrence).

\section{Methods}

\subsection{Study Oversight and Design}

After approval by legal administrative authorities (Comité de Protection des Personnes Est-IV, and Agence Nationale de Sécurité du Médicament, both in 2016), this study was performed in two university-affiliated departments of either medical critical care ( 30 beds) or surgical (18 beds) critical care, and in one medical critical care department of a thirdlevel referral hospital (22 beds). Details of the ALBALSACE study are available at Clinicaltrials.gov: NCT02755155 [19].

\subsection{Patient Selection (Inclusion and Exclusion Criteria)}

As indicated in Figure 1, 128 consecutive adults who were admitted to participating ICUs with shock (acute circulatory failure and lactate $>2 \mathrm{mmol} / \mathrm{L}$ ) requiring the infusion of norepinephrine after correction of hypovolemia with saline, and developing subsequently acute serum albumin $\leq 20 \mathrm{~g} / \mathrm{L}$ were screened for treatment allocation. Written informed consent was obtained for all included patients according to an emergency consent by a next of kin (and confirmed $a$ posteriori by the patient himself in case of survival). The inclusion criteria were diagnosis of acute circulatory failure from suspected septic origin with mean arterial pressure $<60$ $\mathrm{mm} \mathrm{Hg}$, ongoing norepinephrine at any dosage to maintain blood pressure as required by guidelines [20], and occurrence of a serum albumin concentration $\leq 20 \mathrm{~g} / \mathrm{L}$. The exclusion criteria were allergy to human albumin, traumatic brain injury, pregnancy, age $<18$ years, chronic hypoalbuminemia (as met in undernutrition or nephrotic syndrome), patients 
under guardianship or curators, and patients given therapeutic albumin in the month preceding inclusion.

\subsection{Study Treatments}

Before inclusion, patients were treated according to early goal-directed therapy and fluid resuscitation was performed as indicated by the attending physician with the exclusion of colloids and albumin. No other drug was forbidden. At inclusion, using a centralized randomization, stratified by center, with blocks of size 8 , we randomly assigned patients to receive either $12.5 \mathrm{~mL} / \mathrm{kg}$ of 4 percent albumin infused continuously over $24 \mathrm{~h}$ (treatment group) or 20 percent albumin intermittently $(100 \mathrm{~mL}$ over $1 \mathrm{~h}$, every $8 \mathrm{~h}$ (control group), from inclusion to the day when norepinephrine was weaned. During the albumin treatment, daily serum albumin concentrations were measured so that serum albumin be maintained not higher than $30 \mathrm{~g} / \mathrm{L}$. Therapeutic albumin was supplied by the pharmacy from each institution, and purchased at LFB Biomedicaments (Les Ulis, France) either as Vialebex ${ }^{R} 200 \mathrm{mg} / \mathrm{mL}$, bottle of $100 \mathrm{~mL}$, or Vialebex ${ }^{R}$ $40 \mathrm{mg} / \mathrm{mL}$, bottle of $500 \mathrm{~mL}$. The rate of norepinephrine infusion was set to maintain mean arterial pressure above $65 \mathrm{~mm} \mathrm{Hg}$, and weaning of this drug was tested hourly. Of importance is the fact that every patient was given antibiotics for a presumed infection on inclusion in both groups. Finally, all patients were under sedation for ventilation support at first albumin.

\subsection{Outcomes Measures}

The primary outcome measure was all causes mortality rates at 28 days after inclusion. Secondary outcome measures were: 1) the length of both circulatory failure (measured as time from starting to stopping norepinephrine) and the length of albumin treatment (measured from first albumin to end of last albumin infusion); 2) the daily SOFA score change from inclusion to norepinephrine weaning; 3) the incidence of care-related colonization and infection within the hospital; 4) the amount of albumin and its tolerance in each group.

\subsection{Baseline Assessment}

We obtained baseline information on age, gender, causes of referral and severity of the disease. The SAPS II was calculated from the worst values in the $24 \mathrm{~h}$ of admission [21] and the SOFA [22] both on day of admission, day of inclusion and throughout the $96 \mathrm{~h}$ thereafter. We recorded the simultaneous lactate and creatinine. For the purpose of this study, we collected - on inclusion and then each 3 days from inclusion to day 28 - blood samples, lower respiratory tract, urines and skin (or abdominal) samples to check the presence of microbes (bacteria, fungi) and to detect care-related infection. Respiratory samples were only obtained as long as intubation was present and/or a bronco-alveolar lavage was required. When in-dwelling catheters were removed, they were sent for microbial culture. Care-related infections were defined as episodes with clinical signs of focal infection occurring at least after two days of inclusion in the protocol, with fever $\left(>38^{\circ} \mathrm{C}\right)$ or hypothermia $\left(<35^{\circ} \mathrm{C}\right)$, increased Creactive protein $(>8 \mathrm{mg} / \mathrm{L}$, or an increase by $50 \%$ of basal value) and the presence of a microbe at the focus of infection; to be definitively considered as a new infection, these episodes required also that antibiotics be adapted, surgery or drainage be performed to obtain improvement of the patient. Colonization was defined as the presence of bacteria or fungi in an expectedly sterile site, in the absence of a simultaneous increase in C-reactive protein and fever. Immune suppression was defined as any condition or treatment capable of decreasing immunity either by alteration of the functioning of neutrophils or lymphocytes.

\subsection{Statistics}

On the basis of the averaged hospital mortality rates of our three ICUs, and of our current rates of colonization and carerelated infections, the calculated sample size was 550 patients (alpha level $=0.05,80 \%$ power, $20 \%$ versus $30 \%$ of mortality at Day 28). The study protocol planned interim analysis every 50 patients. All analyses were conducted on an intention-to-treat basis. Data are presented as medians [IQR] or as percentages (\%). The between-group mean or percentage difference or relative risk (RR) are given with their $95 \%$ credibility interval and the probability that the value is larger in the 4 percent Albumin group than in the 20 percent albumin group (Pr). A Pr value near 1 indicates a larger value in the 4 percent group and a $\operatorname{Pr}$ value near 0 indicates a smaller value. Repeated data are analyzed with mixed models and count data with Poisson models. Parameters estimates are given with the $95 \%$ credibility interval.

For all analyses, the prior distribution were defined before the study and were based on mild assumptions derived from expert knowledge. In the interim analysis, we computed the predictive probability [23] of superiority of albumin 4 percent group over the 20 percent group. We remind that Bayesian methods do not use $\mathrm{p}$-values and that $\operatorname{Pr}$ (see above) and the predictive probability must not be confused with pvalues. All computations were done with R 3.5.3 and JAGS (Just Another Gibbs Sampler) 4.3.0.

\subsection{Role of the Funding Source}

The trial was funded by a competitive peer-reviewed grant from the Direction de la Recherche Clinique of our universitary hospital (Appel à Projets Internes-2016: Grant n ${ }^{\circ} 6371$ ).

The funder had no role in study design, data collection, data analysis, data interpretation, or writing the report. The corresponding author had full access to all the data in the study and final responsibility for the decision to submit for publication.

\section{Results}

From January 2017 to December 2018, as indicated in the flow diagram of the study (Figure 1), 128 patients (out of 4777 patients screened on admission in the participating 
ICUs) were randomly assigned to receive either continuous 4 percent albumin $(12.5 \mathrm{~mL} / \mathrm{kg}$ over $24 \mathrm{~h}, \mathrm{n}=64)$ or intermittent 20 percent albumin $(100 \mathrm{~mL}$ over $1 \mathrm{~h} / 8 \mathrm{~h}, \mathrm{n}=64)$. Three patients, who were screened but did not receive any treatment, were excluded because of consent withdrawal. Therefore the intention-to-treat analysis included 63 patients in the 4 percent group and 62 patients in the control group.

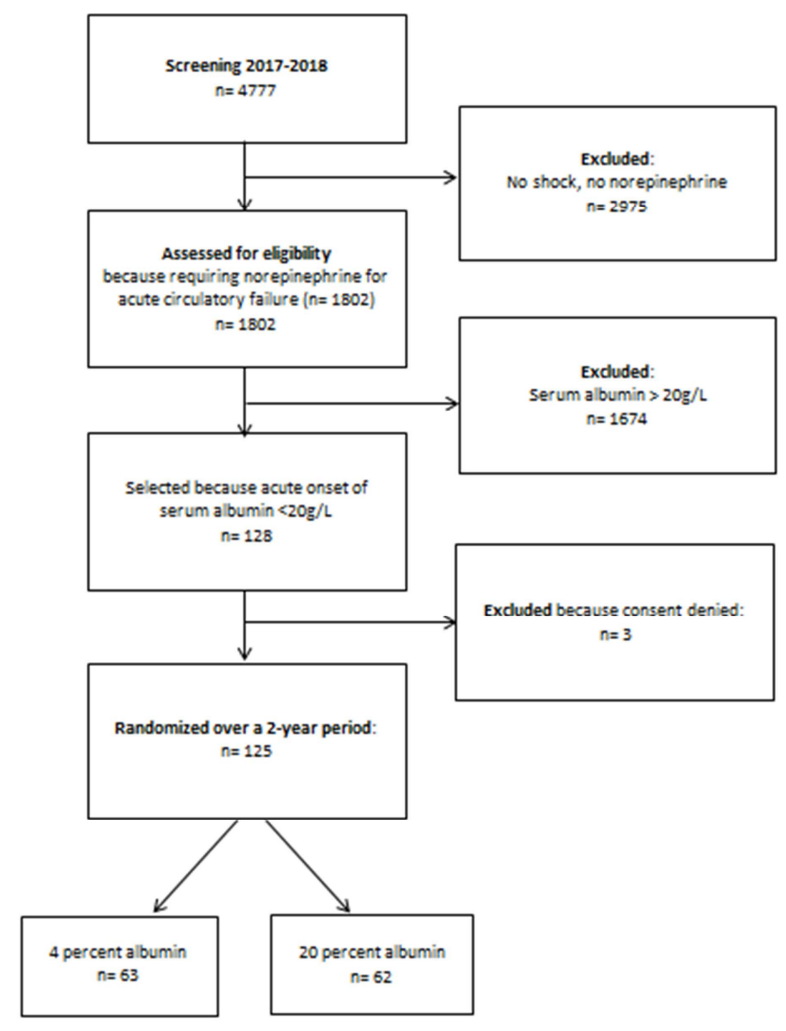

Figure 1. Flow diagram of the progress of the study.

Follow up: there were neither lost to follow-up nor discontinued intervention. Patients analyzed: $n=125$.

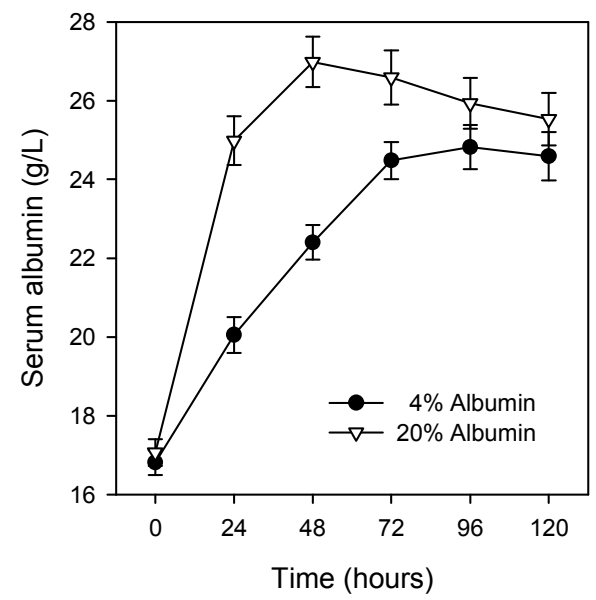

Figure 2. Time-dependent serum albumin concentrations during albumin infusion. To complete see p. 9.

At ICU admission, demographic, clinical, and laboratory features were balanced between the two groups (Table 1), although admission category was more frequently medical than surgical. There were more chronic renal insufficiency patients in the 20 percent albumin group $(\mathrm{Pr}=0.02)$ (Table 1$)$, and patients with solid cancer in remission $>1$ year were more represented in the 4 percent albumin group $(\operatorname{Pr}=0.99)$ (Table 1). At inclusion, the proportion of septic shock patients with positive microbial samples was significantly higher in the 20 percent group than in the 4 percent group $(\mathrm{Pr}=0.001)$ (Table 1).

The length of norepinephrine-infusion time was similar in both groups and so was the time length from norepinephrine treatment starting to the infusion of the first albumin (Table 2).

There was no group differences as far as duration from first albumin to peak of norepinephrine infusion is concerned (Table 2). Neither the duration from admission to first albumin infusion nor the duration from first albumin to last albumin was different between groups (Table 2). The median mass of albumin infused per patient was similar in both groups, but the volume of infusion was increased in the 4 percent albumin group (Table 2). Although the calculation of the treatment time by albumin - assessed from first albumin to last albumin infusion - is similar in both groups (Table 2), it must be kept in mind that the duration of exposition to nonoxidized albumin corresponding to the time interval from first albumin to last albumin in the 4 percent group, is only represented by $1 \mathrm{~h}$ of infusion per bottle of 20 percent albumin in the control group: this leads to a shorter period of fresh albumin availability in this group (Table 2).

The intermittent infusion of 20 percent albumin was associated with a rapid increase in serum albumin concentrations in the range of $25-30 \mathrm{~g} / \mathrm{L}$ until day 4 when the serum albumin levels became similar in the two groups (Figure 2). At that time, according to the study protocol and data in Table 2, the infusion of norepinephrine had been stopped in both groups.

During the 4-day period following inclusion, there was no difference between groups in either SOFA decrease (time $\mathrm{x}$ treatment interaction $0.70[-0.25 ; 1.64], \operatorname{Pr}=0.93$, Figure 3 ), in the plasma creatinine decrease (time $x$ treatment interaction 8.83 [-10.2; 27.7], $\operatorname{Pr}=0.82$, data not illustrated), or in the lactate decrease (time $\mathrm{x}$ treatment interaction 0.13 [$0.85 ; 1.11], \operatorname{Pr}=0.60$, Figure 4 ).

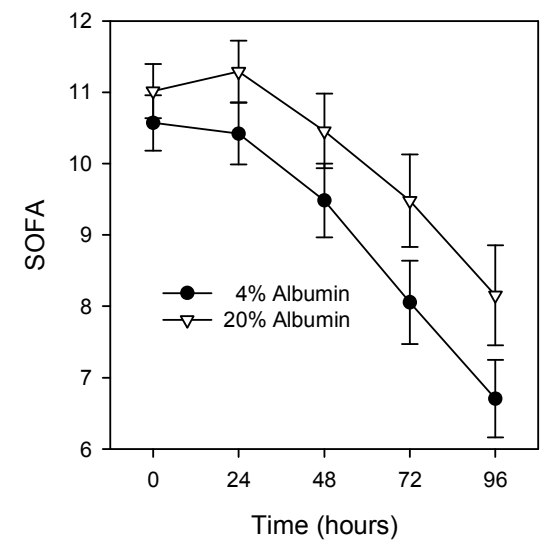

Figure 3. SOFA changes under treatment from inclusion to H96. 


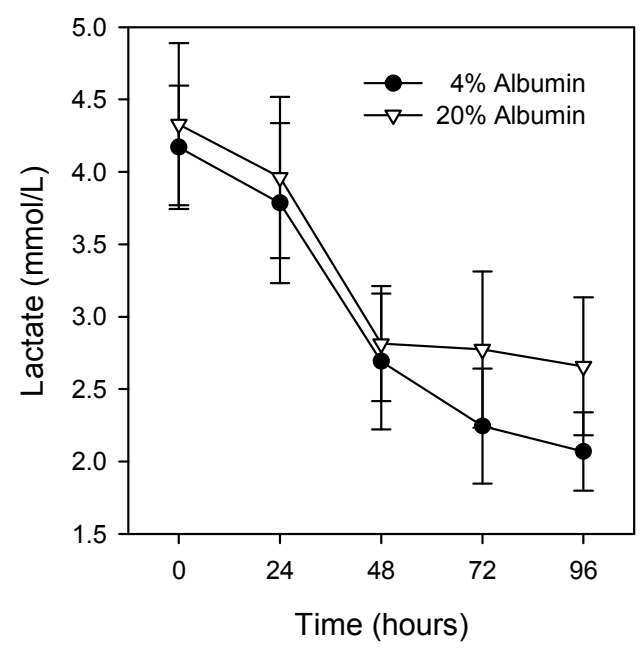

Figure 4. Lactate changes from inclusion to resolution of shock (weaning of norepinephrine, H96).

As far as colonization is concerned, there was no difference between groups $(36.5 \%$ in the 4 percent group versus $46.8 \%$ in the 20 percent group $(\operatorname{Pr}=0.12$, Figure 5 Panel a). However, there was a right shift of the occurrence of colonization in the 4 percent group during the ICU stay as shown in Figure 5 Panel $b(R R=0.86$ [0.47-1.43], $\mathrm{Pr}=0.252)$.

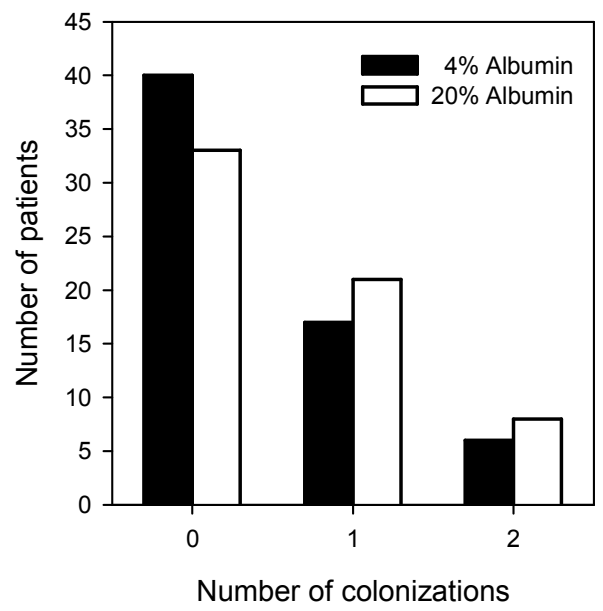

(a)

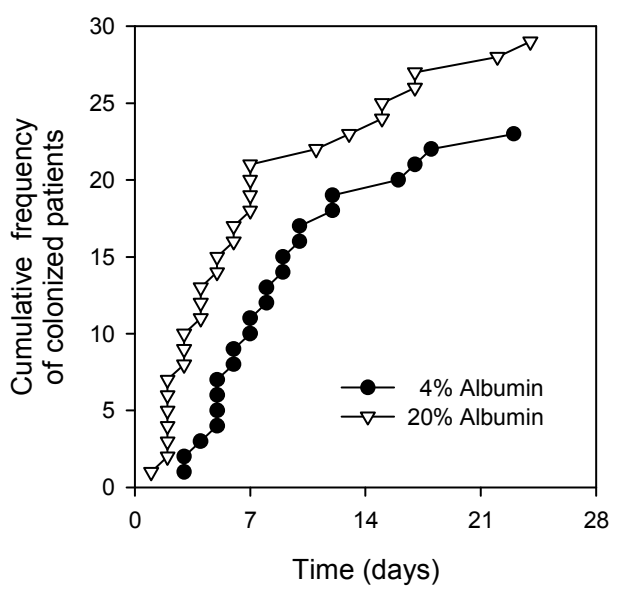

(b)

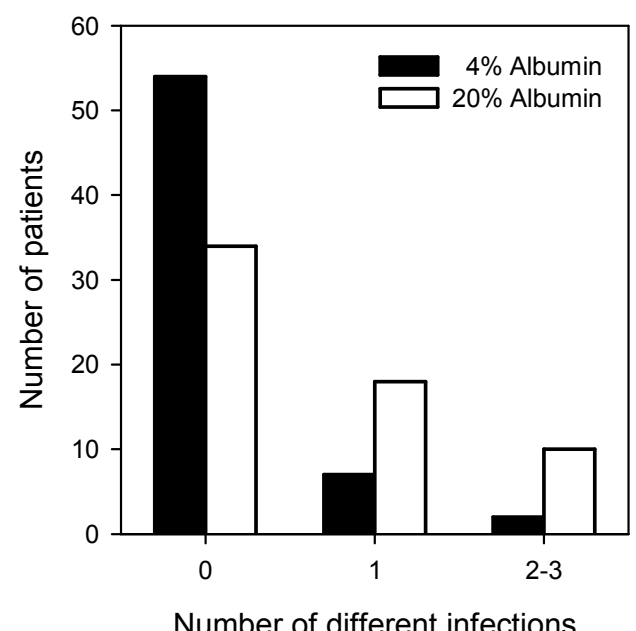

(c)

Figure 5. Occurrence of colonization and of care-related infections in the study population. To complete see p. 9.

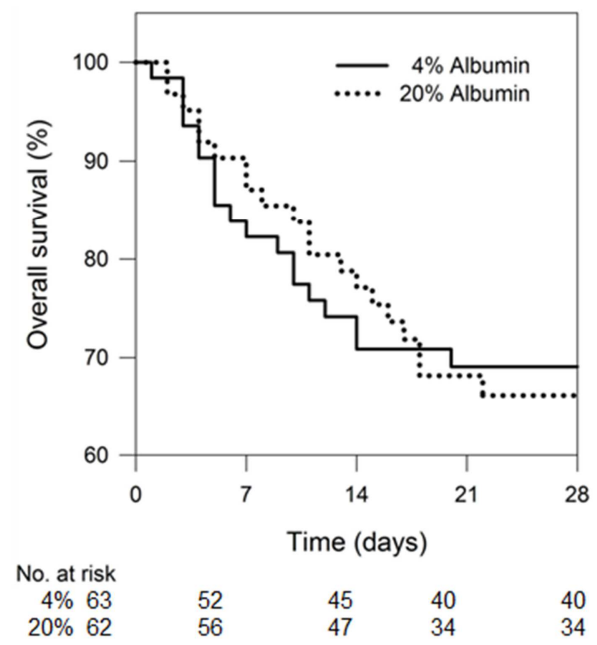

Figure 6. Overall survival to Day 28. To complete see p. 9, 10.

In addition, there were fewer subjects with nosocomial infections in the 4 percent group than in the 20 percent group ( $14.3 \%$ versus $45.2 \%, \operatorname{Pr}<0.001$, Figure 5 Panel c) with a smaller number of nosocomial infections between groups ( $R R=0.36$ [0.18-0.61], $\operatorname{Pr}<0.001)$. The characteristics of nosocomial infections are summarized in Table 3. Bayesian predictive probability at interim analysis showed that the probability of a higher infection rate in the 20 percent albumin group by the end of the study is $>0.999$, with a risk difference estimated to be $29.9 \%$ [14.8-44.6], $\mathrm{Pr}>0.999$.

The 28-day all-cause mortality rate was similar in both group (Table 4, $\mathrm{Pr}=0.40$ ) and so was further mortality at Day28 using the Kaplan-Meier estimates for the probability of overall survival in the intention-to-treat population (Figure 6 ). The Bayesian predictive probability analysis ruled out the possibility that the treatment by 4 percent albumin may reach the main objective at the final 550 inclusion-target, suggesting that the current study be stopped for futility (the risk difference is $2.0 \%[-17.9,14.0]$ and the predictive probability to conclude to a larger death rate in the 20 percent 
Francis Schneider et al: : Continuous 4 Percent Albumin Versus Intermittent 20 Percent Albumin in Adults with Septic Shock: A Prospective, Phase IV, Open-label Randomized Trial

group on final sample target is only 0.402 .

Table 1. Characteristics of the patients.

\begin{tabular}{|c|c|c|c|}
\hline At ICU admission & $4 \%$ Albumin $(n=63)$ & $20 \%$ Albumin $(n=62)$ & $\operatorname{Pr}$ \\
\hline Age - yr & $68.0[58.5-75.5]$ & $68.0[58.2-74.0]$ & 0.58 \\
\hline Male gender - no. (\%) & $41(65.1)$ & $37(59.7)$ & 0.73 \\
\hline Body Mass Index & $26.0[23.1-29.6]$ & $25.2[23.2-30.6]$ & 0.71 \\
\hline \multicolumn{4}{|l|}{ Admission category - no. $(\%)$} \\
\hline Medical & $39(62)$ & $53(85.5)$ & 0.001 \\
\hline Surgical (emergency surgery) & $24(38)$ & $9(14.5)$ & \\
\hline SAPS II score & $58.0[50.0-70.8]$ & $60.0[50.2-75.0]$ & 0.30 \\
\hline SOFA & $9.0[6.0-12.5]$ & $9.0[7.0-12.0]$ & 0.16 \\
\hline Arterial lactate & $3.1[1.9-5.1]$ & $2.7[1.9-4.8]$ & 0.53 \\
\hline Albumin $(g / L)$ & $18.0[15.5-18.5]$ & $17.5[16.0-19.0]$ & 0.23 \\
\hline \multicolumn{4}{|l|}{ Presence of shock no. (\%) } \\
\hline Yes & $61(96.8)$ & $56(90.3)$ & 0.92 \\
\hline No & $2(3.2)$ & $6(9.7)$ & \\
\hline \multicolumn{4}{|l|}{ Comorbidities - no. (\%) } \\
\hline Pulmonary diseases (COPD, asthma, OAS, miscellaneous) & 18 (28.6) & $25(40.3)$ & 0.08 \\
\hline Chronic heart diseases (coronary heart disease, left ventricular failure, arrhymia...) & $31(49.2)$ & $12(19.4)$ & $>0.99$ \\
\hline Hypertension & $35(55.5)$ & $40(64.5)$ & 0.16 \\
\hline Metabolic diseases (diabetes, hypercholesterolemia, thyroïd dysfunctioning...) & $38(60.3)$ & $36(58.1)$ & 0.60 \\
\hline Chronic renal failure $(<30 \mathrm{~mL} / \mathrm{min})$ & $5(7.9)$ & $13(21.0)$ & 0.02 \\
\hline $\begin{array}{l}\text { Malignant hemopathy: } \\
\text { no aplasia }\end{array}$ & $7(11.1)$ & $4(6.5)$ & 0.81 \\
\hline with aplasia & $7(11.1)$ & $10(16.1)$ & 0.21 \\
\hline Solid cancer (remission > 1yr) & $6(9.5)$ & $0(0)$ & 0.99 \\
\hline Recent cancer ( $<1$ yr; no aplasia) & $17(27.0)$ & $9(14.5)$ & 0.96 \\
\hline Immunosuppressive therapy & $6(9.5)$ & $13(21.0)$ & 0.04 \\
\hline Cirrhosis & $11(17.5)$ & $14(22.6)$ & 0.24 \\
\hline \multicolumn{4}{|l|}{ At study inclusion } \\
\hline SOFA & $10[8-13]$ & 11 [9-13] & 0.21 \\
\hline Albumin $(g / L)$ & $18.0[15.5-18.5]$ & $17.5[16-19]$ & 0.29 \\
\hline Serum creatinine $(\mu \mathrm{mol} / \mathrm{L})$ & $127.0[80.0-183.5]$ & $114[73.5-182.0]$ & 0.88 \\
\hline Arterial lactate $(\mathrm{mmol} / \mathrm{L})$ & $2.7[1.8-5.5]$ & $2.5[1.7-5.4]$ & 0.41 \\
\hline \multicolumn{4}{|l|}{ Cause of shock no. (\%) } \\
\hline Septic shock with positive microbial samples & $39(61.9)$ & $51(82.3)$ & 0.001 \\
\hline Septic shock without positive microbial samples & $14(38.1)$ & $11(17.2)$ & \\
\hline
\end{tabular}

Data are medians [IQR] or frequency (\%). SOFA=Sequential Organ Failure Assessment; SAPS II: Simplified Acute Physiologic Score two. To complete see p. 9.

Table 2. Characteristics of study treatments (albumin and norepinephrine).

\begin{tabular}{llll}
\hline & 4 percent albumin $(\mathbf{n}=\mathbf{6 3})$ & 20 percent Albumin $(\mathbf{n}=\mathbf{6 2})$ & Pr \\
\hline Duration of norepinephrine support $(\mathrm{h})$ & $68[50-141]$ & $78[37.5-138.5]$ & 0.87 \\
Time from NE starting to first albumin $(\mathrm{h})$ & $16.8[8.2-28.8]$ & $18.8[6.0-29.0]$ & 0.57 \\
$1^{\text {st }}$ Albumin to peak of NE (length of time, $\left.\mathrm{h}\right)$ & $-3.8[-11.8-4.9]$ & $-2[-13-4.0]$ & 0.27 \\
Admission to $1^{\text {st }}$ albumin (length of time, $\left.\mathrm{h}\right)$ & $23.5[11.2-37.8]$ & $24[13-51.1]$ & 0.21 \\
Duration of albumin infusion (h) & $68.5[46.5-97.2]$ & $56[24.0-112.0]$ & 0.92 \\
Volume of albumin infused (mL/patient) & $4000[2000-6000]$ & $1000[600-1600]$ & 1 \\
Total albumin mass infused (g/patient) & $160[80-240]$ & $200[120-320]$ & 0.61 \\
Albumin exposure per hour of treatment $(\mathrm{g} / \mathrm{h})$ & $2.3[1.8-3.1]$ & $3.8[2.7-6.0]$ & 0.001 \\
Duration of fresh albumin exposure $(\mathrm{h})$ & $68.5[46.5-97.2]$ & $10.0[6.0-16.0]$ & $>0.999$ \\
\hline
\end{tabular}

Data are medians $[\mathrm{IQR}]$ or frequency $(\%)$.

SOFA: Sequential Organ Failure Assessment; SAPS II: Simplified Acute Physiologic Score two. NE: norepinephrine. To complete see p. 9.

Table 3. Care-related infections according to the arm of treatment (4 percent versus 20 percent albumin).

\begin{tabular}{llll}
\hline $\begin{array}{l}\text { 4 percent or 20 } \\
\text { percent Albumin }\end{array}$ & Site of infection & Species involved & $\begin{array}{l}\text { Length of time from first } \\
\text { albumin to infection (days) }\end{array}$ \\
\hline 4 & Blood & S. epidermidis & 12 \\
4 & Blood & S. epidermidis \& C. albicans & 7 \\
4 & Blood & S. epidermidis & 12 \\
4 & Surgical site (abdomen) & E. faecium & 18 \\
4 & Blood \& lower respiratory tract & E. coli \& E. faecium & 12 \\
4 & PAP & C. aeruginosa \& Imipenem R-A. baumanii & 3 \\
4 & Lower respiratory tract & C. glabrata, C. C. albicikeium \& L. mucosa & 5 \\
4 & Surgical site (abdomen) & & 8 \\
\hline
\end{tabular}




\begin{tabular}{|c|c|c|c|}
\hline $\begin{array}{l}4 \text { percent or } 20 \\
\text { percent Albumin }\end{array}$ & Site of infection & Species involved & $\begin{array}{l}\text { Length of time from first } \\
\text { albumin to infection (days) }\end{array}$ \\
\hline 4 & Blood & S. aureus & 12 \\
\hline 20 & Blood & C. glabrata & 4 \\
\hline 20 & VAP and Blood & K. oxytoca, E. faecium \& C. albicans & 17 \\
\hline 20 & Urine & E. faecalis \& H. alvei & 24 \\
\hline 20 & Blood & C. parapsilosis & 14 \\
\hline 20 & Blood stream and lower respiratory tract & P. aeruginosa \& S. maltophilia & 18 \\
\hline 20 & Surgical site (biliary tract) & E. faecium \& S. epidermidis & 7 \\
\hline 20 & Surgical site (abdomen) & E. faecium \& E. aerogenes & 8 \\
\hline 20 & Blood & S. aureus & 28 \\
\hline 20 & Lower respiratory tract & S. epidermidis & 3 \\
\hline 20 & VAP & P. aeruginosa & 7 \\
\hline 20 & Surgical site (biliary tract) & E. cloacae \& S. epidermidis & 3 \\
\hline 20 & VAP & E. coli & 15 \\
\hline 20 & VAP & $P$. aeruginosa & 3 \\
\hline 20 & Lower respiratory tract & S. haemolyticus & 11 \\
\hline 20 & Urine & S. maltophilia & 7 \\
\hline 20 & VAP & E. coli, E. faecalis \& Methicilin-R S. aureus & 8 \\
\hline 20 & Surgical site (abdomen) & E. faecalis & 15 \\
\hline 20 & VAP \& urine & E. cloacae, P. maltophilia \& P. aeruginosa & 7 \\
\hline 20 & Blood \& catheter & C. albicans & 6 \\
\hline 20 & VAP & Pseudomonas aeruginosa \& C. freundii & 4 \\
\hline 20 & VAP & P. aeruginosa & 7 \\
\hline 20 & VAP & S. marcescens & 25 \\
\hline 20 & VAP & E. faecalis \& C. freundii & 4 \\
\hline 20 & VAP & E. faecalis & 24 \\
\hline 20 & Surgical site (abdomen) & K. oxytoca \& E. cloacae & 2 \\
\hline 20 & Surgical site (abdomen) & S. aureus & 4 \\
\hline 20 & VAP & E. faecalis \& E. coli & 4 \\
\hline 20 & Lower respiratory tract & C. argentoratense \& H. simplex virus & 4 \\
\hline 20 & Blood \& catheter & Methicilin Resistant S. aureus & 20 \\
\hline
\end{tabular}

$\mathrm{VAP}=$ ventilator-associated pneumonia were diagnosed using bronco-alveolar lavage fluid analysis and X-Ray changes. To complete see p. 9.

Table 4. Outcomes.

\begin{tabular}{llll}
\hline & 4 percent Albumin $(\mathbf{n}=\mathbf{6 3})$ & 20 percent Albumin $(\mathbf{n}=\mathbf{6 2})$ & Pr \\
\hline Status at 28 days (n, \%) & $19(30.2)$ & $20(32.3)$ & 0.40 \\
Dead & $9(14.3)$ & $13(21.0)$ & 0.17 \\
Alive but still in ICU (n, \%) & $44(69.8)$ & $42(67.7)$ & 0.60 \\
Alive in hospital & $12[7.5-22.0]$ & $13[8.0-24.5]$ & 0.23 \\
Duration of ICU stay (days) & $29[10.5-44.0]$ & $24[14.0-46.8]$ & 0.32 \\
Duration of hospital stay (days) & & & \\
Care-related infections & $23(36.5)$ & $29(46.8)$ & 0.12 \\
Colonization (number of patients) & $9(14.3)$ & $28(45.2)$ & $<0.001$ \\
Care-related infections (number of patients) & & \\
\hline
\end{tabular}

Data are medians $[\mathrm{IQR}]$ or frequency $(\%)$.

ICU: intensive care unit. To complete see p. 9.

\section{Discussion}

In this randomized trial involving real-life practice critically ill patients with septic shock (including patients with aplasia), and developing thereafter acute hypoalbuminemia, the continuous infusion of 4 percent albumin was associated with less care-related infections when compared to intermittent 20 percent albumin. No impact on in-hospital survival or tolerance to albumin was detected.

The most seriously critically ill patients have increased mediators of oxidant stress and a higher incidence of both multiple organ failure and nosocomial infections [24]. The occurrence of the latter is associated with increased morbidity, costs of treatments and with the emergence of threatening bacteria and fungi that are resistant to available antimicrobial drugs [25]. To improve outcome in such patients in addition to conventional precautions, we hypothesized that the restoration of the endogenous functional status of proteins belonging to innate defense would benefit most importantly the ICU-patients with the lowest serum albumin which are prone to care-related infections [26]. Reversing the oxidative stress-related loss of activity of such proteins by anti-oxidant albumin is possible in vitro as far as antimicrobial peptides are concerned with very low amounts of albumin [18]. In vivo, to restore total plasma antioxidant capacity [27] an adequate concentration ratio between the oxidized substrate and the anti-oxidant agent has to be achieved $[17,28]$. Accordingly, we performed this study comparing the continuous supply of non-oxidized 
4 percent albumin [28] with that of intermittent albumin during the acute phase of septic shock which is a model of severe oxidative stress. In our study, the serum albumin concentrations achieved in the experimental arm were constantly lower than in the control arm before the end of acute circulatory failure (which can be dated at the moment when norepinephrine was weaned). Before this trial, we had performed an in vitro assessment of the best concentration of albumin capable of displaying an efficient anti-oxidative power in vivo on oxidized Vasostatin-I, an endogenous antimicrobial peptide: this pilot dose-finding study suggested a possible effect in the range of $10-15 \mathrm{~mL} / \mathrm{kg}$ of 4 percent albumin per day, which additionally excluded harmful sideeffects with reference to recommendation for albumin prescription in humans [18].

In our patients, ICU-mortality and medium term mortality are in the range of those of other septic shock populations in the present era [23, 29-30], and even lower than expected from both the SAPS II score, the admission SOFA and the comorbidities (which included many pathophysiological conditions with at least one immune defect). For long times, the question of why these patients who additionally displayed low serum albumin concentrations have been at high risk of developing care-related infection has not been answered satisfactorily [31]. Our results prove that the continuous infusion of 4 percent albumin was not able to modify mortality when compared to 20 percent albumin. This had also no impact on daily SOFA changes, which suggests the absence of effect on acute circulatory failure, and on each and every organ failure included in this score as well. Finally, lactate decreased to normal range without study groupdifferences suggesting that both albumin treatments impacted similarly on global organ perfusion. Thus, our data are in agreement with meta-analyses that indicate that the therapeutic supply by albumin for the maintenance of a properly working circulation can poorly be recommended to improve mortality [1-3]. Similarly, 4 percent albumin did impact neither on ICU nor on hospital length of stay as previously suggested $[1,3]$.

In contrast, our experimental treatment impacted tangibly on nosocomial infection-related morbidity. Although there was no impact of 4 percent albumin on the number of patients getting colonized during the hospital stay, we noted a difference between 4 percent and 20 percent albumin-treated patients as far as time of colonization is concerned. Microbes-related events occurred as if either 4 percent albumin delays the occurrence of colonization or as 20 percent albumin shortens the time of colonization onset; furthermore, this is occurring within the ICU, not later, most possibly because systematic samplings of microbes was performed rigorously in the ICU - in each and every site of potential infection - whereas it was no longer performed in the airways after ICU-discharge. A decreased rate of early colonization cannot be explained in one arm of treatment by broad spectrum antibiotics given at the acute phase of septic shock because this treatment was present in all patients at inclusion. Thus, our observations are in accordance with our working hypothesis and suggest that a pharmacological manipulation of innate defense altered by endogenous stressrelated oxidation is possible.

A handful of studies suggest the existence of a positive effect of albumin on infection rate lowering in selected cohorts of patients $[10,32]$. The mechanism (s) by which the 4 percent albumin is associated with less infection than 20 percent is, remains unknown. Rarely, albumin has been reported as an antimicrobial agent with antifungal property related to its structure [33]; also, less colonization by fungi is reported to lower the risk of care-related infections [34]. However, the very rare disorder "congenital analbuminemia" has remarkably mild signs and symptoms at all ages without any association with recurrent infections [35]. Therefore, it seems reasonable to propose that a functional and/or indirect mechanism rather than an intrinsic property of albumin may explain our data on the lessening of care-related infection incidence. Posttranscriptional changes of oxidized serum albumin are independently associated with bacterial infections in patients with cirrhosis [36]; in this study, ischemia-modified albumin is reported as elevated in patients with cirrhosis and hyperdynamic circulation without shock [36] and that such an alteration is also associated with bacterial infections. More recently, Caraceni et al. confirmed in vivo that a long-term, low dose albumin administration in decompensated cirrhosis is associated with a significant decrease in spontaneous bacterial infection of ascites but also in other infections, thus confirming the possibility that less albumin than required to normalize the serum concentration range is good for the prevention of infection (32).

The question of why hyperoxidized albumin does not further protect patients as native albumin does has also been addressed in severe alcoholic hepatitis patients who often undergo systemic inflammation: in fact, such albumin modulates neutrophils to further induce oxidative stress and inflammation with a subsequent increased risk of overwhelming bacterial infections (37). Conversely, the effect of albumin on infection prevention whatever the type or origin of infection has never been assessed as a primary objective in a clinical trial involving critically ill patients: more specifically, no study has ever compared 4 percent albumin versus 20 percent albumin probably because a consistent rationale for such a strategy was lacking. Proven in further studies, this concept of treatment may be interesting for large populations of patients at risk of systemic inflammation whether acute or chronic [38].

Our trial has limitations. The most common is that health care professionals were aware of the treatment assignments in agreement with the protocol of infusion. The lack of blinding may have improved the attendance to hygiene rules and thereby lowered the incidence of nosocomial infections, although we did not observe such a trend in other patients treated simultaneously in our ICUs. To compensate for this risk, we chose deliberately to include patients with severe underlying comorbidities (ie: post-chemotherapy aplasia or immunosuppressive drugs for transplantation) which are spontaneously at high risk of nosocomial infection. The real 
impact of the lack of blinding on estimated treatment effects has however recently been questioned and may be less important than previously thought [39]. Finally, our study was stopped on the a planned interim analysis because 1) Bayesian statistics on the primary endpoint suggested futility and 2) for ethical issues based on a secondary endpoint improvement which was not expected to be of such an importance when starting the trial.

\section{Conclusions}

In critically ill patients undergoing an acute oxidative stress who also display recent-onset serum albumin concentrations of $20 \mathrm{~g} / \mathrm{L}$ or lower, a strategy of early and continuous infusion of 4 percent albumin at anti-oxidant dosage is associated with less care-related infection, but it does not result in a mortality rate decrease at 28 days. Large randomized clinical trials are required to validate our findings in other oxidative stress-related disorders before changing clinical practice in other settings.

Caption Figure 2: Serum albumin changes over 120 hours of treatment either by 4 percent albumin (black circles) or by 20 percent albumin (white triangles). Data are means +/SEM.

Caption Figure 5: The proportion of colonization was similar in the two arms of treatment by albumin (Panel a, Pr $(4 \%>20 \%)=0.12)$. As indicated in Panel $b$, the cumulative frequency of colonized patients was shifted rightwards in the 4 percent albumin treated patients when compared to controls. Care-related infections were significantly decreased in the 4 percent albumin group (Panel c) when compared to the controls $(\operatorname{Pr}(4 \%>20 \%)<0.001)$.

Caption Table 1: Bayesian methods do not use p-values; $\mathrm{Pr}$ must not be confused with p-values. Two values of Pr are of interest: value near 1 for the 4 percent group and the value near 0 for the 20 percent group.

Caption Table 2: The third column corresponds to the posterior probability that group 4 is higher than group 20, for the mean or percentage difference. Bayesian methods do not use p-values; Pr must not be confused with p-values. Two values of $\operatorname{Pr}$ are of interest: value near 1 for the 4 percent group and the value near 0 for the 20 percent group.

Caption Table 4: Bayesian methods do not use p-values; $\mathrm{Pr}$ must not be confused with p-values. Two values of Pr are of interest: value near 1 for the 4 percent group and the value near 0 for the 20 percent group.

Caption Table 3: Lower respiratory tract infections were diagnosed using tracheal fluid aspiration and ventilatorassociated pneumonia using bronco-alveolar lavage. Blood stream infection was diagnosed using commercially available culture vials (BD Bactec ${ }^{\mathrm{TM}}$, USA). Positive catheter culture was defined by a catheter tip culture performed with the Brun-Buisson technique yielding $\geq 103$ colony-forming units $/ \mathrm{mL}$. Sampling on operative sites was performed by surgeons within the operating theater. In a few instances, infection has been related to fungi because only these microbes were found in situ, and also because antifungal drugs were necessary to full recovery of the patient: this cannot rule out that the focus of infection could have been triggered by a viral species (see for instance patient 7 ).

Caption Figure 6: Kaplan-Meier estimates for the probability of overall survival in the intention-to-treat population. Dotted line $=20$ percent albumin treated patients; black line $=4$ percent albumin-treated patients. There was no difference in survival time within 28 days after inclusion.

\section{Supplementary Informations}

\section{Funding}

Direction de la Recherche Clinique, Hôpitaux Universitaires de Strasbourg, API-2016 Grant n 6371.

\section{Clinical Trial}

\section{NCT02755155.}

\section{Conflict of Interest}

None declared.

\section{Declaration of Interests}

None.

\section{Author Contributions}

FS designed the protocol, was study chief investigator, reviewed the data, including interpretation of the results and co-wrote the manuscript. VC and GM ran the study at the Hautepierre I hospital, and made intellectual input in the study protocol and manuscript writing. A-F D and A P ran the study at the Mulhouse hospital (GHMSA), whereas Pierre-Olivier Ludes ran the study at the Hautepierre II hospital. BS and TF checked the data and performed statistical analyses, NM co-designed the study protocol, designed the statistical plan, supervised the analyses, and cowrote the manuscript. M-H M-B co-wrote the study protocol and the manuscript, reviewed the data, including interpretation of the results.

\section{Complete List of Sub-investigators}

Service de Médecine Intensive Réanimation, Hôpital de Hautepierre I, Hôpitaux Universitaires de Strasbourg, France (ARTZNER Thierry, BALDACINI Mathieu, FAGOTGANDET Florence, GAUDEFROY Julie, GUILLOT Max, HARLAY Marie-Line, HERBRECHT Jean-Etienne, JANSSEN-LANGENSTEIN Ralf, LACAN Claire, LAVIGNE Thierry, LEROUX Justine, MAESTRAGGI Quentin, MICHARD Baptiste, SCHENCK Maleka).

Service d'Anesthésie-Réanimation chirurgicale Hôpital de Hautepierre II, Hôpitaux Universitaires de Strasbourg, France (BRAUN Thierry, DHIF Nadia, DIEMUNSCH Pierre, POTTECHER Julien, RAMEAU Jean-Pierre, SZCZOT Magdalena).

Service des Urgences médico-chirurgicales Hôpital de 
Hautepierre I, Hôpitaux Universitaires de Strasbourg, France (LEBORGNE Pierrick).

Service de Pharmacie, Hôpital de Hautepierre I, Hôpitaux Universitaires de Strasbourg, France (LEVEQUE Dominique).

Service de Médecine Intensive Réanimation, Groupe Hospitalier Mulhouse Sud Alsace, Mulhouse, France (GUIOT Philippe, IONESCU Carmen, KUTEIFAN Khaldoun).

\section{Acknowledgements}

The study was supported by the Direction de la Recherche Clinique, Hôpitaux Universitaires de Strasbourg (France) for the financial support («Appel à Projets Internes 2016» grant 6371). The authors thank Mrs Parvin Mahoney for editing the English manuscript, the study patients and their relatives for their participation and the physicians and nursing staff of the participating centers for their cooperation. We also acknowledge the help of Sylvie L'Hotellier, Michel Masuchio in data collection.

\section{References}

[1] Alderson P, Bunn F, Li Wan Po A, Li L, Blackhall K, Roberts I and Schierhout G. Human albumin solution for resuscitation and volume expansion in critically ill patients. Cochrane Database Syst Rev. 2011 Oct 5; (10): CD001208. doi: $10.1002 / 14651858$

[2] Patel A, Laffan MA, Waheed U and Brett SJ. Randomized trials of human albumin for adults with sepsis: systematic review and meta-analysis with trial sequential analysis of allcause mortality. BMJ. 2014 Jul 22; 349: g4561. doi: 10.1136/bmj.g4561.

[3] Jiang L, Jiang S, Zhang M, Zheng Z and Ma Y. Albumin versus other fluids for fluid resuscitation in patients with sepsis: a meta-analysis. PLoS One 2014 Dec 4; 9 (12): e114666. doi: 10.1371/journal.pone.0114666.

[4] Roche M, Rondeau P, Singh NR, Tarnus E and Bourdon E. The antioxidant properties of serum albumin. FEBS Lett. 2008; 582: 1783-1787. doi: 10.1016/j.febslet.2008.04.057.

[5] Ospina-Tascon G, Neves AP, Occhipinti G, Donadello K, Büchele G, Simion D, Chierego ML, Silva TO, Fonseca A, Vincent JL and De Backer D. Effects of fluids on microvascular perfusion in patients with severe sepsis. Intensive Care Med. 2010; 36: 949-955. doi: 10.1007/s00134010-1843-3.

[6] Caironi P, Tognoni G, Masson S, Fumagalli R, Pesenti A, Romero M, Fanizza C, Caspani L, Faenza S, Grasselli G, Lapichino G, Antonelli M, Parrini V, Fiore G, Latini R and Gattinoni L. ALBIOS Study Investigators. Albumin replacement in patients with severe sepsis or septic shock. N Engl J Med. 2014 Apr 10; 370 (15): 1412-1421. doi: 10.1056/NEJMoa1305727.

[7] Uhlig C, Silva PL, Deckert S, Schmitt J and de Abreu MG. Albumin versus crystalloid solutions in patients with the acute respiratory distress syndrome: a systematic review and metaanalysis. Crit Care. 2014 Jan 9; 18 (1): R10. doi: 10.1186/cc13187.
[8] Wiedermann CJ, Dunzendorfer S, Gaioni LU, Zaraca F and Joannidis M. Hyperoncotic colloids and acute kidney injury: a meta-analysis of randomized trials. Crit Care. 2010; 14 (5): R191. doi: 10.1186/cc9308.

[9] Myburgh J, Cooper DJ, Finfer S, Bellomo R, Norton R, Bishop N, Kai Lo S and Vallance S. SAFE Study Investigators; Australian and New Zealand Intensive Care Society Clinical Trials Group; Australian Red Cross Blood Service; George Institute for International Health. Saline or albumin for fluid resuscitation in patients with traumatic brain injury. N Engl J Med. 2007 Aug 30; 357 (9): 874-884. doi: 10.1056/NEJMoa067514.

[10] Guevara M, Terra C, Nazar A, Solà E, Fernández J, Pavesi M, Arroyo $\mathrm{V}$ and Ginès P. Albumin for bacterial infections other than spontaneous bacterial peritonitis in cirrhosis. A randomized, controlled study. J Hepatol. 2012 Oct; 57 (4): 759-765. doi: 10.1016/j.jhep.2012.06.013.

[11] Finfer S, Bellomo R, Boyce N, French J, Myburgh J and Norton R. The SAFE Study Investigators. A comparison of albumin and saline for fluid resuscitation in the intensive care unit. N Engl J Med. 2004 May 27; 350 (22): 2247-2256. doi: 10.1056/NEJMoa040232.

[12] Domenicali M, Baldassarre M, Giannone FA, Naldi M, Mastroroberto M, Biselli M, Laggetta M, Patrono D, Bertucci C, Bernardi M and Caraceni P. Posttranscriptional changes of serum albumin: clinical and prognostic significance in hospitalized patients with cirrhosis. Hepatology. 2014 Dec; 60 (6): 1851-1860. doi: 10.1002/hep.27322.

[13] Jalan R, Schnurr K, Mookerjee RP, Sen S, Cheshire L, Hodges S, Muravsky V, Williams R, Matthes G and Davies NA. Alterations in the functional capacity of albumin in patients with decompensated cirrhosis is associated with increased mortality. Hepatology. 2009 Aug; 50 (2): 555-564. doi: 10.1002/hep.22913.

[14] Alonso de Vega JM, Díaz J, Serrano E, Carbonell LF. Oxidative stress in critically ill patients with systemic inflammatory response syndrome. Crit Care Med. 2002 Aug; 30 (8): 1782-1786. doi: 10.1097/00003246200208000-00018.

[15] Naldi M, Baldassarre M, Domenicali M, Bartolini M and Caraceni P. Structural and functional integrity of human serum albumin: Analytical approaches and clinical relevance in patients with liver cirrhosis. J Pharm Biomed Anal. 2017 Sep 10; 144: 138-153. doi: 10.1016/j.jpba.2017.04.023.

[16] Watanabe H, Imafuku T, Otagiri M and Maruyama T. Clinical implications associated with the posttranslational modification-induced functional impairment of albumin in oxidative stress-related diseases. J Pharm Sci. 2017 Sep; 106 (9): 2195-2203. doi: 10.1016/j.xphs.2017.03.002.

[17] Halliwell B and Whiteman M. Measuring reactive species and oxidative damage in vivo and in cell culture: how should you do it and what do the results mean? Br J Pharmacol. 2004 May; 142 (2): 231-255. doi: 10.1038/sj.bjp.0705776.

[18] Schneider F, Dureau, AF, Hellé, S, Betscha C, Senger B, Cremel G, Boulmedais F, Strub, JM, Corti A, Meyer N, Guillot M, Schaaf P and Metz-Boutigue MH. A pilot study on continuous infusion of $4 \%$ albumin in critically ill patients: impact on nosocomial infection via a reduction mechanism for oxidized substrates. Crit Care Expl 2019; 1: e0044. doi: 10.1097/CCE.000000000000044. 
[19] https://clinicaltrials.gov/ct2/show/NCT02755155?term=al bumine + and + strasbourg\& cond $=$ Hypoalbuminemia\&rank $=1$.

[20] Rhodes A, Evans LE, Alhazzani W, Levy MM, Antonelli M, Ferrer R, Kumar A, Sevransky JE, Sprung CL, Nunnally ME, Rochwerg B, Rubenfeld GD, Angus DC, Annane D, Beale RJ, Bellinghan GJ, Bernard GR, Chiche JD, Coopersmith C, De Backer DP, French CJ, Fujishima S, Gerlach H, Hidalgo JL, Hollenberg SM, Jones AE, Karnad DR, Kleinpell RM, Koh Y, Lisboa TC, Machado FR, Marini JJ, Marshall JC, Mazuski JE, McIntyre LA, McLean AS, Mehta S, Moreno RP, Myburgh J, Navalesi P, Nishida O, Osborn TM, Perner A, Plunkett CM, Ranieri M, Schorr CA, Seckel MA, Seymour CW, Shieh L, Shukri KA, Simpson SQ, Singer M, Thompson BT, Townsend SR, Van der Poll T, Vincent JL, Wiersinga WJ, Zimmerman JL and Dellinger RP. Surviving Sepsis Campaign: International Guidelines for Management of Sepsis and Septic Shock: 2016. Intensive Care Med 2017; 43: 304-377. doi: 10.1007/s00134-017-4683-6.

[21] Le Gall JR, Lemeshow S and Saulnier F. A new Simplified Acute Physiology Score (SAPS II) based on a European/North American multicenter study. JAMA. 1993; 270: 2957-2963. doi: 10.1001/jama.270.24.2957.

[22] Vincent JL, Moreno R, Takala J, Willatts S, De Mendonça A, Bruining H, Reinhart C K, Suter PM and Thijs LG. The SOFA (Sepsis-related Organ Failure Assessment) score to describe organ dysfunction/failure. On behalf of the Working Group on Sepsis-Related Problems of the European Society of Intensive Care Medicine. Intensive Care Med. 1996; 22: 707-710. doi: $10.1007 / \mathrm{bf01709751.}$

[23] Lee JJ and Liu DD. A predictive probability design for phase II cancer clinical trials. Clin Trials. 2008; 5: 93-106. doi: 10.1177/1740774508089279.

[24] Van Vught LA, Klein Klouwenberg PMC, Spitoni C, Scicluna BP, Wiewel MA, Horn J, Schultz MJ, Nürnberg P, Bonten MJM, Cremer OL, Van der Poll T and MARS Consortium. Incidence, risk factors, and attributable mortality of secondary infections in the intensive care unit after admission for sepsis. JAMA. 2016; 315: 1469-1479. doi: 10.1001/jama.2016.2691.

[25] Timsit J F, Esaied W, Neuville M, Bouadma L and Mourvllier B. Update on ventilator-associated pneumonia F1000Res. 2017: 6: 2061 . doi: 10.12688/f1000research.12222.1.

[26] Hennessey DB, Burke JP, Ni-Dhonochu T, Shields C, Winter DC and Mealy K. Preoperative hypoalbuminemia is an independent risk factor for the development of surgical site infection following gastrointestinal surgery: a multiinstitutional study. Ann Surg. 2010; 252: 325-329. doi: 10.1097/SLA.0b013e3181e9819a.

[27] Quinlan GJ, Mumby S, Martin GS, Gordon RB, Gutteridge JMC and Evans TW. Albumin influences total plasma antioxidant capacity favorably in patients with acute lung injury. Crit Care Med. 2004; 32: 755-759. doi: 10.1097/01.ccm.0000114574.18641.5d.

[28] Marie AL, Przybylski C, Gonnet F, Daniel R, Urabin R, Chevreux G, Jorieux $\mathrm{S}$ and Taverna M. Capillary zone electrophoresis and capillary electrophoresis-mass spectrometry for analyzing qualitative and quantitative variations in therapeutic albumin. Anal Chim Acta. 2013; 800:
103-110. doi: 10.1016/j.aca.2013.09.023.

[29] Contou D, Roux D, Jochmans S, Coudroy R, Guérot E, Grimaldi D, Ricome S, Maury E, Plantefève G, Mayaux J, Dessap AM, Brun-Buisson C and de Prost N. Septic shock with no diagnosis at 24 hours: a pragmatic multicenter prospective cohort study. Critical Care. 2016; 20: 360. doi: 10.1186/s13054-016-1537-S.

[30] Quenot JP, Binquet C, Kara F, Martinet O, Ganster F, Navellou JC, Castelain V, Barraud D, Cousson J, Louis G, Perez P, Kuteifan K, Noirot A, Badie J, Mezher C, Lessire H and Pavon A.The epidemiology of septic shock in French intensive care units: the prospective multicenter cohort EPISS study. Crit Care. 2013 Apr 25; 17 (2): R65. doi: 10.1186/cc12598.

[31] Domínguez de Villota E, Mosquera JM, Rubio JJ, Galdos P, Díez Balda V, de la Serna JL and Tomás MI. Association of a low serum albumin with infection and increased mortality in critically Ill patients. Intensive Care Med. 1980; 7: 19-22. doi: $10.1007 / \mathrm{bf} 01692917$.

[32] Caraceni P, Riggio O, Angeli P, Alessandria C, Neri S, Foschi FG, Levantesi F, Airoldi A, Boccia S, SvegliatiBaroni G, Fagiuoli S, Romanelli RG, Cozzolongo R, Di Marco V, Sangiovanni V, Morisco F, Toniutto P, Tortora A, De Marco R, Angelico M, Cacciola I, Elia G, Federico A, Massironi S, Guarisco R, Galioto A, Ballardini G, Rendina M, Nardelli S, Piano S, Elia C, Prestianni L, Cappa FM, Cesarini L, Simone L, Pasquale C, Cavallin M, Andrealli A, Fidone F, Ruggeri M, Roncadori A, Baldassarre M, Tufoni M, Zaccherini G, Bernardi M; ANSWER Study Investigators. Long-term albumin administration in decompensated cirrhosis (ANSWER): an open-label randomized trial. Lancet. 2018 Jun 16; 391 (10138): 2417-2429. Erratum in: Lancet. 2018 Aug 4; 392 (10145): 386. doi: 10.1016/S01406736(18) 30840-7.

[33] Arzumanyan VG, Ozhovan IM and Svitich OA. Antimicrobial Effect of Albumin on Bacteria and Yeast Cells. Bull Exp Biol Med. 2019 Oct; 167 (6): 763-766. doi: 10.1007/s10517-01904618-6.

[34] Roux D, Gaudry S, Khoy-Ear L, Aloulou M, PhillipsHoulbracq M, Bex J, Skurnik D, Denamur E, Monteiro RC, Dreyfuss D and Ricard JD. Airway fungal colonization compromises the immune system allowing bacterial pneumonia to prevail. Crit Care Med. 2013; 41: e191-9. doi: 10.1097/CCM.0b013e31828a25d6.

[35] Koot BG, Houwen R, Pot DJ and Nauta J. Congenital analbuminaemia: biochemical and clinical implications. A case-report and literature review. Eur J pediatr. 2004; 163: 664-670. doi: 10.1007\%2Fs00431-004-1492-z.

[36] Giannone FA, Domenicali M, Baldassarre M, Bartoletti M, Naldi M, Laggetta M, Bertucci C, Colecchia A, Viale P, Bernardi $\mathrm{M}$ and Caraceni $\mathrm{P}$. Ischaemia-modified albumin: a marker of bacterial infection in hospitalized patients with cirrhosis. Liver Int. 2015; 35: 2425-2432. doi: 10.1111/liv.12860.

[37] Das S, Maras JS, Hussain MS, Sharma S, David P, Sukriti S, Shasthry SM, Maiwall R, Trehanpati N, Singh TP and Sarin SK. Hyperoxidized albumin modulates neutrophils to induce oxidative stress and inflammation in severe alcoholic hepatitis. Hepatology. 2017; 65: 631-641. doi: 10.1002/hep.28897. 
[38] Boada M, Ortiz P, Anaya F, Hernández I, Muñoz J, Núñez L, Olazarán J, Roca I, Cuberas G, Tárraga L, Buendia M, Pla RP, Ferrer I and Páez A. Efficacy and safety of plasma exchange with $5 \%$ albumin to modify cerebrospinal fluid and plasma amyloid-B concentrations and cognition outcomes in Alzheimer disease patients: a multicenter, randomized, controlled clinical trial. J Alzheimer Dis. 2017; 56: 129-143. doi: 10.1358/dnp.2009.22.6.1395256.
[39] Moustgaard H, Clayton GL, Jones HE, Boutron I, Jørgensen L, Laursen DRT, Olsen MF, Paludan-Müller A, Ravaud P, Savović J, Sterne JAC, Higgins JPT and Hróbjartsson A. Impact of blinding on estimated treatment effects in randomized clinical trials: meta-epidemiological study. BMJ. 2020; 368: 16802. Erratum in: BMJ. 2020 Feb 5; 368: m358. doi: 10.1136/BMJ; 16802. 